IMAGE CHANGE DETECTION USING WIRELESS SENSOR NETWORKS

\begin{abstract}
A Thesis by
Sree Ramya Yelisetty

B.TECH in Electronics and Communications Engineering

J.B. Institute of Engineering \& Technology, India, 2004.

Submitted to the Department of Electrical Engineering and the faculty of the Graduate School of Wichita State University

in partial fulfillment of the requirements for the degree of Master of Science
\end{abstract}

May 2007 


\section{IMAGE CHANGE DETECTION USING WIRELESS SENSOR NETWORKS}

I have examined the final copy of this thesis for form and content and recommend that it be accepted in partial fulfillment of the requirements for the degree of Master of Science, with a major in Electrical Engineering.

Kamesh Namuduri, Committee Chair

We have read this thesis and

recommend its acceptance:

Hyuck Kwon, Committee Member

Alexandre Boukhgueim, Committee Member 


\section{DEDICATION}

To my loving parents, brother, and my beloved husband 


\section{ACKNOWLEDGEMENTS}

It is with pleasure that I convey my gratitude to all the people who were important to the successful realization of my thesis. You all have played indispensable roles in my life and work. I thank you from my heart.

First, I thank my advisor Dr. Kamesh Namuduri for his advice, supervision, and guidance throughout my studies at Wichita State University. Above all, he provided me with unflinching encouragement and support in various ways. He has always been my source of inspiration. I am grateful for him showing me the way to this research. In addition to being an excellent supervisor, he was as close as a relative and a good friend. Dr. Namuduri, I thank you once again for all your support, understanding, and encouragement.

I also thank Dr. Hyuck M. Kwon and Dr. Alexandre Boukhgueim for sparing their time and for providing opinions on my thesis work.

I would be nowhere without the support of my family. I feel lucky to have such a family. They never said no to any of my requests. They taught me how important it is for a girl to be independent and to work hard to meet goals. My mother has been my source of inspiration. She is a wonderful woman who balanced the tremendous duties of a mother and an industrialist. My father played a key role in embedding in my childhood the right to a fundamental education. I thank you dear mom and dad for all your support and your belief in me. I am also grateful to my brother for all his support and encouragement.

The most important person behind this achievement is my husband. His love, encouragement, support, and sacrifice helped me to successfully complete this degree. I also thank my in-laws for their support and encouragement.

I would like to thank my friends Neelu, Shilpa, Harini, Dipti, Upul, Anil, and many 
others who supported me during my stay at Wichita State University. I also thank my friends Priyanka, Pratima, Praveena, and Swetha for their encouragement. And I thank Gowrav for listening to all my problems during my research.

Again, thank you all so much. 


\begin{abstract}
Change detection in images is of great interest because of its relevance in many applications such as video surveillance. This thesis work presents the underlying theoretical problem of distributed image change detection using a wireless sensor network. The proposed system consisted of multiple image sensors, which made local decisions independently and sent them to a base station through an additive white Gaussian noise channel. The base station then made a global decision and declared whether a significant change had occurred or not.

This system used four thresholds to detect local and global changes in the area being monitored. Two thresholds defined at the sensor level helped the sensor make a local decision, and the remaining two thresholds defined at the system level helped the fusion center make a global decision. Hence, by using four thresholds, the performance of the proposed model was observed to have very good fault tolerance.
\end{abstract}




\section{TABLE OF CONTENTS}

Chapter

1. INTRODUCTION AND PREVIEW .1

1.1 Image Change Detection and Its Applications.........................................................

1.2 Distributed Signal Processing, Distributed Networks, and Distributed Detection .....2

1.3 Distributed Image Change Detection and Its Applications.........................................2

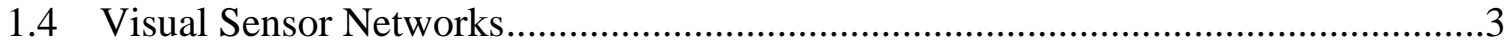

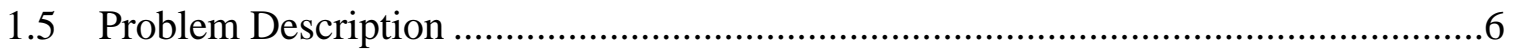

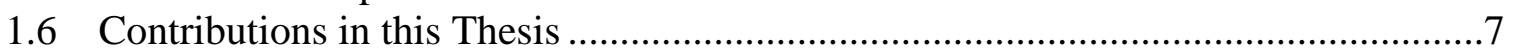

1.6.1 Local Change Detection..........................................................................

1.6.2 Global Change Detection ......................................................................

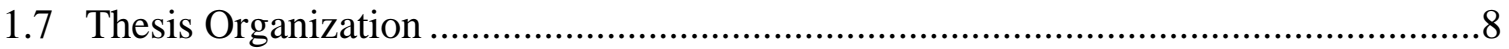

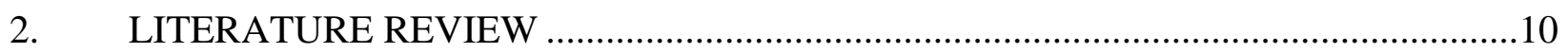

2.1 Change Detection............................................................................................10

2.2 Image Change Detection...................................................................................

2.3 Applications of Image Change Detection .................................................................11

2.4 Distributed Detection and Decision Fusion …………................................................13

2.5 Visual Sensors and Visual Sensor Networks ..........................................................15

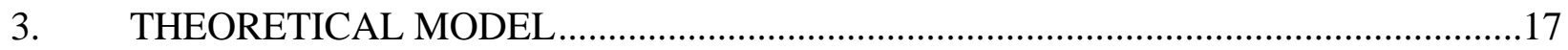

3.1 Processing at the Sensors ................................................................................17

3.2 Processing at the Fusion Center ............................................................................23

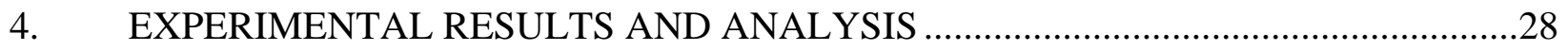

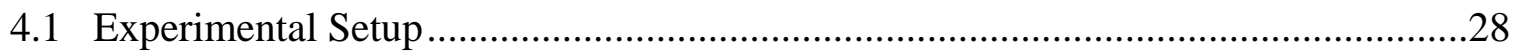

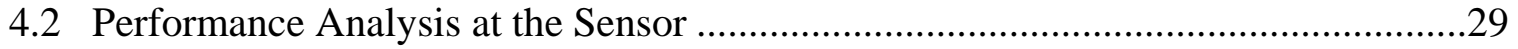

4.3 Performance Analysis at the Fusion Center....................................................................34

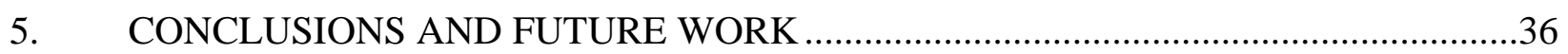

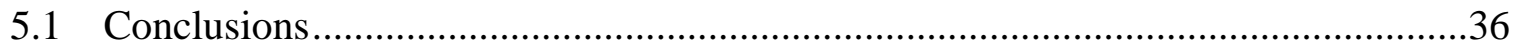

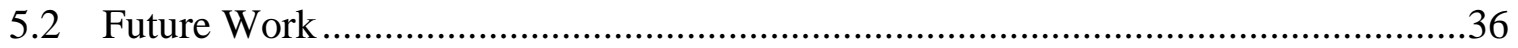

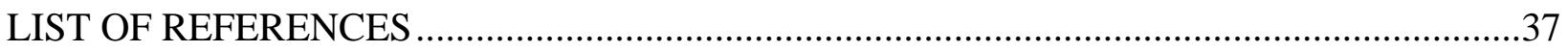




\section{LIST OF FIGURES}

Figure

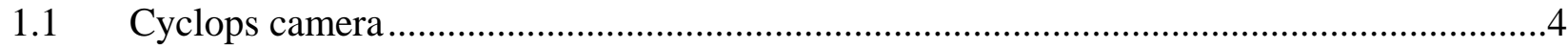

1.2 Two images captured through Cyclops camera .........................................................5

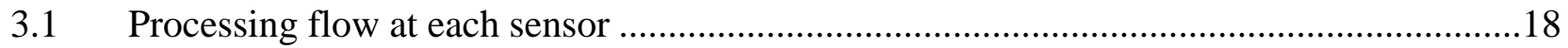

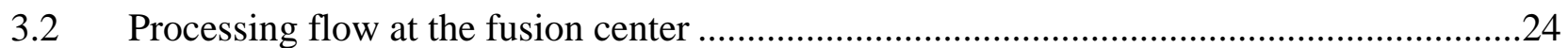

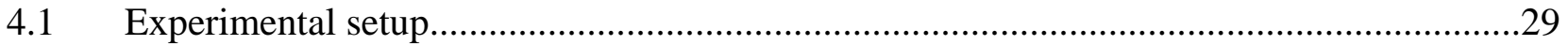

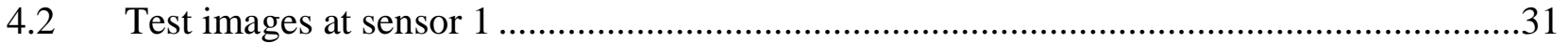

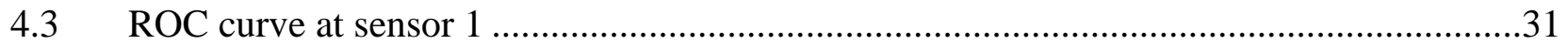

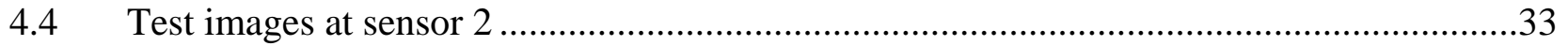

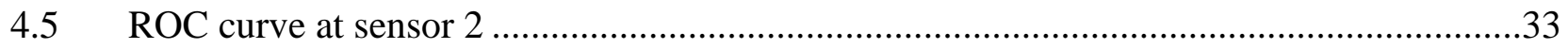

4.6 ROC curves at fusion center with 2, 3, and 4 local detections ......................................35 


\section{Chapter 1}

\section{Introduction and Preview}

\subsection{Image Change Detection and Its Applications}

Change detection in multiple images of the same scene taken at different times is of significant interest because of its relevance in a number of applications, such as video surveillance, medical diagnosis, and remote sensing [1].

Video surveillance is the most important application among those mentioned above. Video surveillance is useful for security enhancement in places such as airports, museums, and shopping malls, which require continuous monitoring. This is an extremely computational intensive task, due to the amount of information that must be processed and transferred within the network. Hence, an automated video surveillance system in commercial and defence applications is greatly needed. Several processes in a full-fledged surveillance system include change detection, coding and communication of image data, local and global decision-making, all related to the significance of events taking place at the scene, decision fusion, and object or target recognition. 


\subsection{Distributed Signal Processing, Distributed Net- works, and Distributed Detection}

Distributed signal processing in a network has several advantages, such as reducing the amount of communication in the network, reducing the risk for node failures, and helping the fusion center from becoming overwhelmed with large quantities of raw data from the nodes. Typically, distributed or collaborative signal processing can be classified into two areas: distributed detection and distributed estimation. This thesis mainly deals with the principles of distributed detection. A distributed network consists of intelligent nodes, which can make local decisions independently and forward them to the fusion center. Since sensors are intelligent devices, the concept of distributed signal processing can be applied to wireless sensor networks. In recent years, more research is being done in decentralized or distributed detection. The best application for distributed detection can be seen in the field of sensor networks. Distributed detection can be performed using three major topologies: parallel, serial or tandem, and tree configurations [2]. Distributed detection in a network consisting of several nodes deals with local processing at each node followed by global processing at the fusion center. Local and global processing gives rise to local and global decisions, which in turn can be made by using Neyman Pearson criterion, Mini-max criterion, or Bayesian criterion.

\subsection{Distributed Image Change Detection and Its Applications}

Distributed image change detection combines the principles of image change detection and distributed detection. Distributed image change detection is well suited 
in applications such as video surveillance using wireless image sensor networks. The concept of distributed detection helps in effective utilization of network resources such as bandwidth and energy in wireless sensor networks. In video surveillance, several image sensors are deployed in the region of interest to monitor and detect significant changes. The decision-making process at sensor level makes use of image change detection principles. The fusion center combines local decisions by making use of decision-fusion principles. This global decision for change detection triggers an alarm and alerts the system if a change is detected in the scene being monitored. An automated video surveillance system can replace the traditional video surveillance system, which requires a large amount of resources and continuous human monitoring.

\subsection{Visual Sensor Networks}

A visual sensor network consists of low-cost and low-energy image sensors, which are deployed in a region of interest to observe a phenomenon and send those observations to a fusion center or base station, which makes a global assessment. As with any sensor network, a visual sensor network also suffers from constraints on resources, such as computational power, bandwidth, and energy. As new applications emerge, it is important to know the fundamental limits of information processing, encoding, fusion, communication, control, and decision-making processes in a visual sensor network. From this perspective, the information theoretic aspects play an important role in visual sensor networks. It is probably due to the feasibility of deploying sensor network platforms, that the theoretical aspects of sensor networks have recently come to the forefront of research.

One of the best examples of a visual sensor is a Cyclops camera. A Cyclops 
camera, depicted in Figure 1.1, is a small low-power color camera designed jointly by Agilent Laboratories and the Center for Embedded Network Sensing at UCLA [3]. Two test images taken from this camera are depicted in Figure 1.2. The camera can be connected to the Crossbow's wireless motes [4]. Crossbow motes (for example, MICA2) are small wireless sensor boards that can be connected to sensors capable of sensing environmental parameters such as temperature, light, and humidity, and transmitting the measured data to other motes or a base station, which are located within their radio transmission range. Motes run the code developed in NesC language [5] on a TinyOS operating system [6].

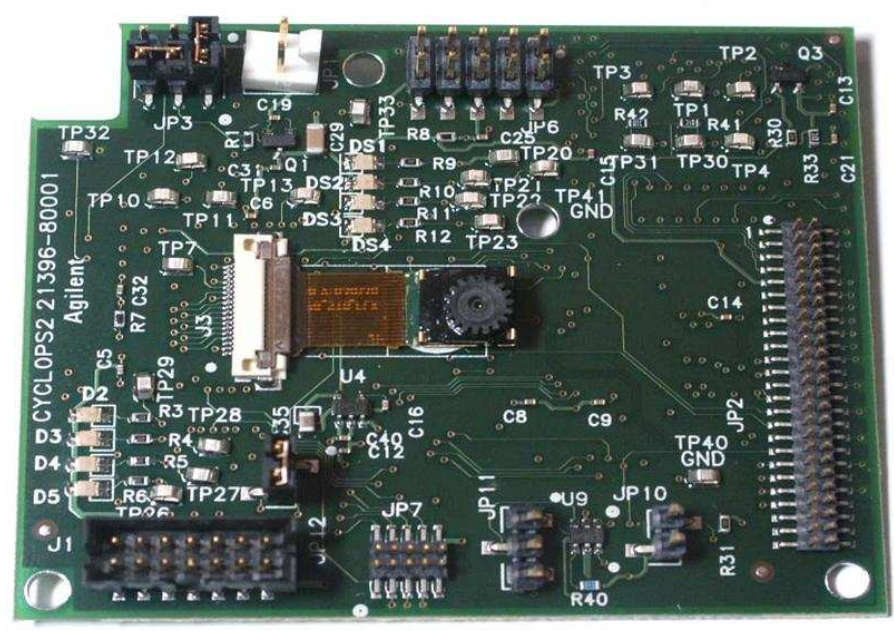

Figure 1.1: Cyclops camera. 

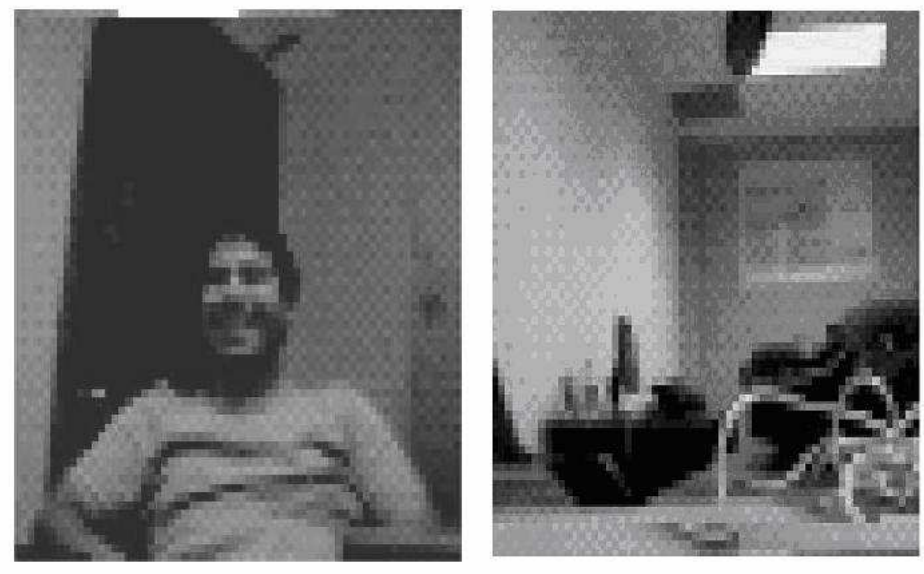

Figure 1.2: Two images captured through Cyclops camera.

This combination can be used to develop several applications over a wireless sensor network. Connecting Cyclops cameras to wireless motes allows the sensor network to acquire images at regular intervals and transmit them to the desktop/laptop over a wireless channel. Such a visual sensor network can be used to extract meaningful information that can be used for object detection, recognition, motion detection, video surveillance, and monitoring applications. Due to limitation on its memory, a Cyclops camera can acquire images of resolution 64x64 pixels only, as opposed to very high-resolution images that normally are obtained by high-end cameras. 


\subsection{Problem Description}

This thesis investigated distributed image change detection using a wireless image sensor network. A wireless image sensor network consists of image sensors or cameras as nodes. These image sensors capture images at fixed intervals of time and relay them to the base station. The main objective of the sensors is to monitor and detect changes in a given region and report these changes to the fusion center. A wireless sensor network typically operates under severe resource constraints (bandwidth, energy, etc). Hence, it is essential to limit the communication between sensors and the fusion center as much as possible. One way to accomplish this is to allow the cameras to perform simple computations and make local decisions. These local decisions are of the form of "1" or "0," indicating change and no change, respectively. These local decisions can be relayed to a fusion center, which makes a global decision by aggregation. The process of aggregating local decisions and global decision-making is called as decision fusion [7] [8].

Several algorithms have been developed for detecting changes in images. The most simple and effective algorithm is image differencing. Image differencing is performed by taking the difference between two images of the same scene at different instances. This leads to the formation of a difference image, which can depict the regions of change in the scene being monitored. Image change can be caused by significant changes such as appearance and disappearance of objects as well as motion of objects, and insignificant changes such as sensor noise, camera motion, atmospheric absorption, and so on [1]. The challenge lies in distinguishing between significant changes and insignificant changes. This is achieved by appropriate selection of four thresholds in such a way that significant changes can be distinguished, which in turn 
helps to improve the system performance. Also, sending raw image data from the sensors to the fusion center increases the use of system resources such as energy and bandwidth. The challenge here lies in reducing the amount of communication between the sensors and the fusion center.

\subsection{Contributions in this thesis}

This thesis investigated the underlying theoretical problems of distributed image change detection in a wireless image sensor network. The system made use of four thresholds to detect local and global changes in the area being monitored. Two thresholds defined at the sensor level helped the sensor make a local decision and the remaining two thresholds were defined at the system level to help the fusion center make a global decision. Transmission of local decisions in the form of binary data, as opposed to directly sending raw image data to a fusion center, may significantly save system resources such as bandwidth, energy, and so on.

\subsubsection{Local Change Detection}

In a distributed wireless image sensor network, sensors were deployed in a random fashion to observe a particular scene or region of interest. Each sensor made its own decision without any communication from its neighboring sensor nodes. The image sensors acquired images at regular intervals and computed image difference. Each pixel in the difference image was compared to the first local threshold say $T_{1}$. The pixels whose intensity exceeded the defined threshold were called active pixels, and those pixels whose intensity did not exceed the threshold were called inactive pixels.

The second threshold, say $T_{2}$, helped in making a decision for change detection at 
the sensor level. This decision rule counted the number of active pixels and compared it with the threshold $T_{2}$. If the count exceeded $T_{2}$, then the image was called an active image and a decision was made for a change, otherwise the image was called an inactive image and a decision was made for no change in the scene being monitored. In this way, a change was detected locally at the sensor level, which formed a local decision at the sensor. These local decisions were of the form of " 1 " for a change and "0" for no change and were relayed to the fusion center through a wireless channel.

\subsubsection{Global Change Detection}

The fusion center, or base station, collected the local decisions from all sensors in the network and made a decision for global change detection. The fusion center first detected the local decisions, which were corrupted due to the addition of noise in the wireless channel, using a threshold $T_{3}$. A fusion rule was then derived for detecting a global change. A fusion rule, which counted the number of local detections was compared to the global threshold, say $T_{4}$. If the number of local detections exceeded the global threshold $T_{4}$, then a global change was detected, otherwise no change was detected. This was the final decision in the system, which alerted the system when a change was detected.

\subsection{Thesis Organization}

This thesis is divided into five chapters. Chapter 1 provides an introduction to image change detection, distributed detection, distributed image change detection, and their applications, such as video surveillance. It also discusses visual sensor networks and finally describes the significance and contribution of this thesis. 
Chapter 2 provides a literature survey in the fields of image change detection, distributed detection, and visual sensor networks.

In Chapter 3, processing at the sensor followed by processing at the fusion center are explained in detail.

In Chapter 4, the experimental setup followed by the results of the proposed system are presented.

Finally, the conclusion and scope of future work are presented in Chapter 5. 


\section{Chapter 2}

\section{Literature Review}

\subsection{Change Detection}

Change detection is the process of identifying differences in the state of an object or phenomenon by observing it at different times. Change detection in images deals with identifying the set of pixels that is significantly different between two consecutive images of the same scene [1]. This identification provides the information about the existence of change and its location. Changes can be caused by appearance and disappearance of objects, movement of objects relative to the background, and shape changes of objects.

\subsection{Image Change Detection and its Importance}

In the literature, image change detection has been investigated in centralized systems [1]. However, decentralized image change detection is a new topic and has not been investigated. After identifying changes in two consecutive images the next step is to determine if the changes are significant or insignificant. Thresholding is 
an important step in distinguishing significant changes from insignificant changes. A framework for testing and evaluating eight different thresholding algorithms for change detection in surveillance environment has been presented by Rosin and Ioannidis [9]. A change detection algorithm that uses image difference with two thresholds (one low and one high) has been developed by Bruzzone and Prieto [10]. If the pixel intensity in the corresponding difference image is greater than the higher threshold, then the corresponding pixel is categorized in a change class. However, if the difference pixel intensity is lower than the lower threshold, then the corresponding pixel is categorized in an unchanged class. The remaining pixels whose difference intensity levels lie between these two thresholds are allowed for further processing, where the spatial-contextual information based on Markov random field model is considered. It is known that intensity levels of neighboring pixels of images have significant correlation, and changes most likely can occur in connected regions rather than disjointed pixels. Using this fact, a more accurate change detection algorithm is built by considering Markov random fields, which exploits the statistical correlation of intensity levels among neighboring pixels [11].

\subsection{Applications of Image Change Detection}

Image change detection has several applications, such as video surveillance, remote sensing, medical diagnosis, and under-water sensing. Video surveillance is one of the important applications of image change detection [12], [13]. The main objective of any video surveillance system is to check for an unusual event in the scene being monitored. An automated video surveillance system includes an intelligent network module that triggers an alarm when unusual events occur. Video surveillance 
encompasses several research fields such as signal processing, image processing, computer vision, communications networking, pattern recognition, sensor networks, and information fusion.

The development of a smart video surveillance system enhances security and situation awareness across multiple scales of space and time. The main aim of a multi-scale tracking system is to gather information about objects in the monitored area at several scales in a unified framework. The concept of a multi-scale tracking system along with the technical challenges involved therein have been presented by Hampapur et al. [14].

A heterogenous video surveillance system consists of fixed and mobile cameras. The combination of cheaper and faster computing hardware with efficient and versatile sensors creates a complex system architecture leading to the development of multicamera or multi-tier systems. Change detection methods in a heterogenous video surveillance system have been presented by Foresti et al. [15].

Automatic change detection also plays an important role in medical applications. An intelligent framework for automatic change detection in serial MRI's has been presented by Bosc et al. [16]. Once a disease is diagnosed, long-term monitoring of disease evolution is essential. By directly comparing successively scanned images, manual detection sometimes turns out to be erroneous because small artifacts might go unnoticeable by the human eye. Hence, this framework of image change detection would help in monitoring and noticing the disease evolution. 


\subsection{Distributed Detection and Decision Fusion}

A sensor network that makes use of distributed detection principles must combine data or decisions from all sensors at the fusion center which in turn makes a final global decision. Data fusion deals with the processing of raw data relayed by sensors at the fusion center, whereas decision fusion deals with processing of local decisions relayed by sensors at the fusion center. Thus, decision fusion can be viewed as a special case of data fusion [17].

Decision fusion allows efficient utilization of energy and bandwidth in the network. A decision fusion rule that fuses the local decisions from the sensors has been proposed [7], [8]. The fact that power received at the sensors is inversely proportional to the square of the distance between the target and the sensors has been used to derive the equations for probability of detection, false alarm, and miss in [7], [8]. The local decisions and fusion rule are derived based on a binary hypothesis testing model. The fusion rule is derived using the total number of detections from local sensors in a wireless sensor network consisting of a large number of sensors.

In real-time applications, there is always a chance that some sensors are out of communication range of the fusion center or run out of battery power. Thus, it makes sense to consider the number of sensors in a wireless sensor network as a random variable. A Poisson random variable has been considered to represent the number of sensors in a network [8].

A distributed sensor network that is subjected to power constraint has been considered [18], [19]. In this model, local processing at the sensors involves amplifying and forwarding the data or observations to the fusion center. The decision rule at the fusion center is derived using Neyman-Pearson and Bayesian criteria. Decentralized 
detection under conditionally independent observations has been studied [18] as well as decentralized detection under independent observations [19].

Decision fusion using hierarchial models has been presented by Niu and Varshney [8], Chen and Varshney [20]. In large sensor networks, the sensors are deployed randomly, and some sensors might be far away from the target. Since the power received at the sensors is inversely proportional to the distance between the target and the sensors, the sensors that are far away from the target receive noisy observations. A hierarchial model is a suitable choice for modeling such situations. In a hierarchial sensor network model, sensors that are close to each other form a cluster, and each cluster has a cluster head. These cluster heads receive data from the clustered nodes and make local decisions, which are then forwarded to the fusion center. Hence, by forming clusters in large sensor networks, noisy transmissions from the sensors can be avoided to some extent.

Three major topologies used for distributed signal processing are parallel, serial or tandem, and tree configurations [2]. It is known that decision rules can be derived by using either Neyman-Pearson, Mini-max, or Bayesian criterion. The distributed detection problem under parallel and serial topologies is tested using Neyman-Pearson and Bayesian criterion.

Typically, distributed detectors are categorized into two types, namely fixedsample size detectors and sequential detectors [21]. Fixed-sample size detectors are those that operate with a fixed number of observations that are predetermined at the time the detector is designed. On the other hand, a sequential detector is one in which the number of observations used in deciding between null or alternate hypothesis is a random variable. 
Quickest change detection is one type of sequential detection that has been studied extensively in the literature [21], [22]. The quickest change detection problem is also called a disorder problem. This problem deals with the detection of an abrupt change in distribution as soon as possible, with some constraints on rate or probability of false alarms.

\subsection{Visual Sensors and Visual Sensor Networks}

A sensor with camera on board is called a visual sensor. The advent of visual sensors led to a new set of applications along with associated challenges. Due to their wide range of applications, such as video surveillance, remote sensing, tracking, and face recognition, visual sensor networks have become very important in recent years. Hierarchial networks or multi-tier systems are recent research topics in this area [23]. A multi-tier system is a network consisting of multiple tiers, where each tier constitutes a different type of sensor. For example, a two-tier system consists of low-resolution camera sensors in the lower tier and high-resolution camera sensors in the higher tier. By making use of these multi-tier systems, several benefits, such as low cost, high reliability, high functionality, and high coverage, can be achieved.

In general, visual sensors consume high power when compared to other sensors such as thermal sensors, pressure sensors, and light sensors. Since images contribute to large amounts of data, the image or visual sensors consume high power. Hence, effective power utilization in real-time applications is very essential. The power utilization in different types of sensors is presented by Kulkarni et al. [23].

Visual sensors and image sensors have a wide range of applications. One such application is concealed weapon detection. The detection of weapons underneath a 
person's clothing from a fair distance before manual screening is desirable. Chen et al. provide a list of imaging sensors used for this purpose, comparing them in terms of illumination, proximity and portability [24].

A visual sensor network is a network consisting of several visual sensors capable of processing and acquiring images from a variety of view points and a base station that can fuse images. A visual sensor network combines the principles of image change detection and distributed detection. Visual sensor networks have opened up new challenges in the research world and novel applications for the industry. In order to develop efficient algorithms and applications using visual sensor networks, a significant amount of research needs to be done in the following areas: distributed image processing, image fusion, global and local image fusion, image communications, source channel coding, image registration, and distributed image registration.

Visual sensor networks are primarily employed for monitoring and surveillance of wide areas. As with any sensor network, a visual sensor network suffers from constraints on resources such as computational power, bandwidth, and energy. The analysis of power consumption and processing time for different tasks in a wireless sensor network have been studied [25]. Based on this analysis, the lifetime of a visual sensor network can be predicted. 


\section{Chapter 3}

\section{Theoretical Model}

\subsection{Processing at the Sensors}

Consider the scenario of a wireless sensor network with $m$ image sensors observing a particular scene. The main objective of the sensor network is to detect significant changes in the scene being monitored. Each image sensor is allowed to take images at fixed intervals of time. $D\left(\mathbf{x}_{i}\right)=\left|\mathrm{f}_{1}\left(\mathbf{x}_{i}\right)-\mathrm{f}_{2}\left(\mathbf{x}_{i}\right)\right|$ represents the difference pixels, $\mathbf{x}_{i}$ represents the pixel coordinate, $\mathrm{f}_{1}\left(\mathbf{x}_{i}\right)$ and $\mathrm{f}_{2}\left(\mathbf{x}_{i}\right)$ represent the intensity value at location $\mathbf{x}_{i}$ in two different images $f_{1}$ and $f_{2}$, and $i=1,2, . . n$ represents the index of the pixel. The processing flow at each sensor is illustrated in Figure 3.1. The set of difference pixels between two different images forms a difference image. This difference image can be considered as an instance of a random vector, with each element or a pixel corresponding to an instance of a random variable. A pixel in the difference image is considered an active pixel if its intensity exceeds a certain threshold; otherwise, it is considered an inactive pixel. The threshold $T_{1}$ is computed based on a binary hypothesis test that results in $1 / 0$ decisions. 


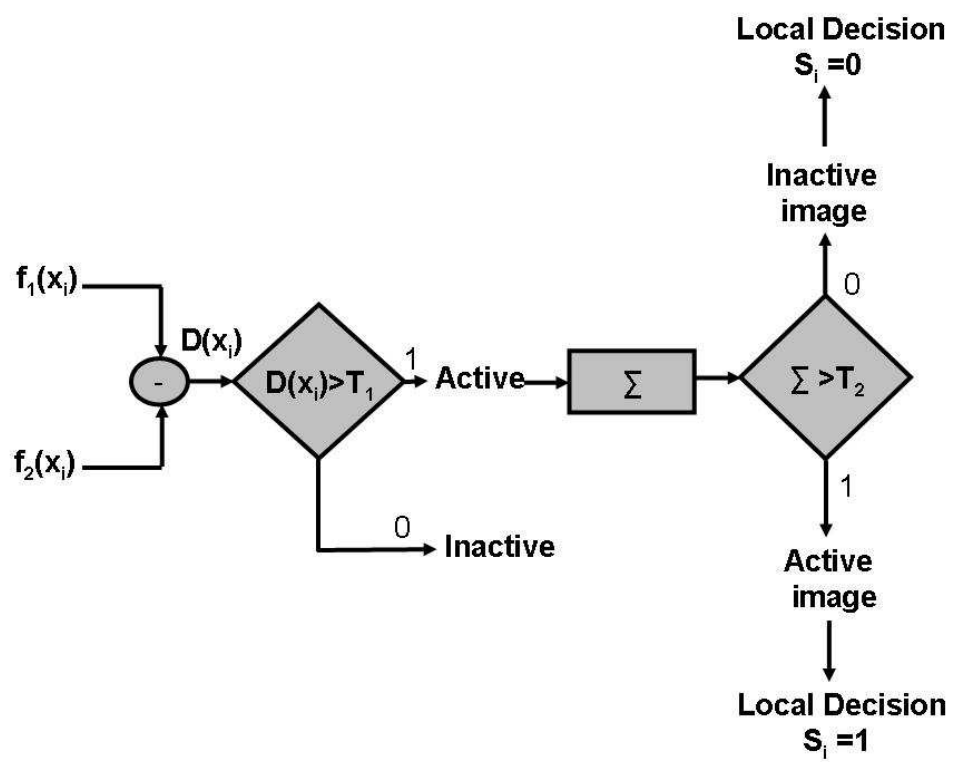

Figure 3.1: Information processing flow at individual sensors. In the first stage, the difference at each pixel in two different images of the same scene is computed and compared with a threshold $T_{1}$. If the difference is greater than $T_{1} 1$ is generated; otherwise 0 is generated. The next step is to count the number of " 1 "s or active pixels and compare it with another threshold $T_{2}$. If the count is greater than the $T_{2}$, 1 is generated signifying an active image; otherwise, 0 is generated which signifies an inactive image. These local decisions $S_{i}$ in the form of $1 / 0$ are transmitted via a wireless channel to the base station.

Suppose $\delta\left(D\left(\mathbf{x}_{i}\right)\right)$ represents the decision at pixel $\mathbf{x}_{i}$. Then the decision rule can be expressed as

$$
\delta\left(D\left(\mathbf{x}_{i}\right)\right)= \begin{cases}1, & \text { if } \mathbf{x}_{i} \text { is active i.e, } D\left(\mathbf{x}_{i}\right)>T_{1} \\ 0, & \text { if } \mathbf{x}_{i} \text { is inactive i.e, } D\left(\mathbf{x}_{i}\right)<T_{1}\end{cases}
$$

Let $p\left(D\left(\mathbf{x}_{i}\right) \mid H_{0}\right)$ and $p\left(D\left(\mathbf{x}_{i}\right) \mid H_{1}\right)$ represent the class conditional density functions of inactive pixels $\left(H_{0}\right)$ and active pixels $\left(H_{1}\right)$, respectively. Assume that both densities 
follow Gaussian distributions, which are defined as

$$
\begin{aligned}
& p\left(D\left(\mathbf{x}_{i}\right) \mid H_{0}\right)=\frac{1}{\sqrt{2 \pi \sigma_{0}^{2}}} \exp \left\{\frac{-\left(D\left(\mathbf{x}_{i}\right)-\mu_{0}\right)^{2}}{2 \sigma_{0}^{2}}\right\} \\
& p\left(D\left(\mathbf{x}_{i}\right) \mid H_{1}\right)=\frac{1}{\sqrt{2 \pi \sigma_{1}^{2}}} \exp \left\{\frac{-\left(D\left(\mathbf{x}_{i}\right)-\mu_{1}\right)^{2}}{2 \sigma_{1}^{2}}\right\}
\end{aligned}
$$

where $\mu_{0}, \mu_{1}, \sigma_{0}^{2}$, and $\sigma_{1}^{2}$ denote the means and variances for the corresponding density functions, respectively.

Based on equations (3.1.2) and (3.1.3), the likelihood ratio can be defined as

$$
L\left(D\left(\mathbf{x}_{i}\right)\right)=\frac{p\left(D\left(\mathbf{x}_{i}\right) \mid H_{1}\right)}{p\left(D\left(\mathbf{x}_{i}\right) \mid H_{0}\right)}=e^{\frac{\mu_{1}-\mu_{0}}{\sigma^{2}}\left\{D\left(\mathbf{x}_{i}\right)-\frac{\mu_{0}+\mu_{1}}{2}\right\}}
$$

assuming that $\sigma_{0}^{2}=\sigma_{1}^{2}=\sigma^{2}$.

Now, the likelihood ratio needs to be compared with a threshold $T_{1}$ to decide whether the change at a given pixel is significant or not. The threshold can be fixed using Bayesian criterion, Neyman-Pearson criterion, or Mini-max criterion. In this thesis, the Bayesian criterion was used to compute the threshold $T_{1}$ which is defined as

$$
T_{1}=\frac{p\left(H_{0}\right)\left(C_{10}-C_{00}\right)}{p\left(H_{1}\right)\left(C_{01}-C_{11}\right)}
$$

where $p\left(H_{0}\right)$ and $p\left(H_{1}\right)$ are the a priori probabilities that are independent of the observations. The elements $C_{10}, C_{00}, C_{01}$ and $C_{11}$ form a cost matrix. The elements $C_{00}$ and $C_{11}$ represent costs incurred in taking correct decisions, i.e, costs incurred in stating inactive and active pixels as inactive and active pixels, respectively. Similarly, the costs $C_{10}$ and $C_{01}$ represent those incurred in taking wrong decisions, i.e, costs incurred in stating inactive and active pixels as active and inactive pixels, respectively. 
Based on equations (3.1.2), (3.1.3), (3.1.4), and (3.1.5), the decision rule can be simplified as

$$
\delta\left(D\left(\mathbf{x}_{i}\right)\right)= \begin{cases}1, & D\left(\mathbf{x}_{i}\right) \geqslant \frac{\left(\mu_{0}+\mu_{1}\right)}{2}+\frac{\sigma^{2} \log \left(T_{1}\right)}{\left(\mu_{1}-\mu_{0}\right)} \\ 0, & D\left(\mathbf{x}_{i}\right)<\frac{\left(\mu_{0}+\mu_{1}\right)}{2}+\frac{\sigma^{2} \log \left(T_{1}\right)}{\left(\mu_{1}-\mu_{0}\right)}\end{cases}
$$

The decision statistic $\left(D\left(\mathbf{x}_{i}\right)\right)$ takes the value 1 when the pixel at location $\mathbf{x}_{i}$ is active and the value 0 when the pixel is inactive.

The next step is to count the number of active pixels within the difference image and compare it with another threshold $T_{2}$. The selection of the threshold $T_{2}$ decides whether a change has occurred locally. If the number of active pixels is greater than the threshold $T_{2}$, then the difference image is considered as an active image; otherwise it is considered as an inactive image.

Assume that the size of the image is $n$, and define a statistic $\lambda_{1}$, which describes the sum of active pixels in the difference image as [7] [8]:

$$
\lambda_{1}=\sum_{i=1}^{n} \delta\left(D\left(\mathbf{x}_{i}\right)\right)
$$

Assume the probabilities for active image or detection as $P_{a i}$, probability of inactive image or rejection as $P_{i i}$, probability of false alarm as $P_{f a}$, and probability of miss as $P_{\text {miss }}$.

The above-mentioned probabilities can be written as, [26]

$$
\begin{aligned}
& P_{i i}=P\left\{\lambda_{1}<T_{2} \mid H_{0}\right\} \\
& P_{a i}=P\left\{\lambda_{1} \geq T_{2} \mid H_{1}\right\} \\
& P_{f a}=P\left\{\lambda_{1} \geq T_{2} \mid H_{0}\right\}
\end{aligned}
$$




$$
P_{\text {miss }}=P\left\{\lambda_{1}<T_{2} \mid H_{1}\right\}
$$

It can be shown that $\lambda_{1}$ follows a Binomial distribution. Let the probabilities of active pixels and inactive pixels in a difference image be represented as $p_{a p}$ and $p_{i p}$, respectively, which are calculated as

$$
p_{a p}=\lambda_{1} / n, \quad p_{i p}=1-p_{a p}
$$

Therefore, for a given threshold $T_{2}$, the probabilities can be calculated as

$$
\begin{gathered}
P_{i i}=\sum_{i=0}^{T_{2}}\left(\begin{array}{c}
n \\
i
\end{array}\right) p_{i p}^{i}\left(1-p_{i p}\right)^{(n-i)} \\
P_{a i}=\sum_{i=T_{2}}^{n}\left(\begin{array}{c}
n \\
i
\end{array}\right) p_{a p}^{i}\left(1-p_{a p}\right)^{(n-i)} \\
P_{f a}=1-P_{i i}=1-\sum_{i=0}^{T_{2}}\left(\begin{array}{c}
n \\
i
\end{array}\right) p_{i p}^{i}\left(1-p_{i p}\right)^{(n-i)} \\
P_{m i s s}=1-P_{a i}=1-\sum_{i=T_{2}}^{n}\left(\begin{array}{c}
n \\
i
\end{array}\right) p_{a p}^{i}\left(1-p_{a p}\right)^{(n-i)}
\end{gathered}
$$

When $n$ is large enough, the probabilities in equations (3.1.13) to (3.1.16) can be calculated by using the Laplace-Demoivre approximation [27] as

$$
\begin{gathered}
P_{i i} \simeq Q\left(\frac{-T_{2} p_{i p}}{\sqrt{n p_{i p}\left(1-p_{i p}\right)}}\right) \simeq 0.5-0.5 \operatorname{erf}\left(\frac{-T_{2} p_{i p}}{\sqrt{2 n p_{i p}\left(1-p_{i p}\right)}}\right) \\
P_{a i} \simeq Q\left(\frac{T_{2}-n p_{a p}}{\sqrt{n p_{a p}\left(1-p_{a p}\right)}}\right) \simeq 0.5-0.5 \operatorname{erf}\left(\frac{T_{2}-n p_{a p}}{\sqrt{2 n p_{a p}\left(1-p_{a p}\right)}}\right) \\
P_{f a} \simeq 1-Q\left(\frac{-T_{2} p_{i p}}{\sqrt{n p_{i p}\left(1-p_{i p}\right)}}\right) \simeq 0.5+0.5 \operatorname{erf}\left(\frac{-T_{2} p_{i p}}{\sqrt{2 n p_{i p}\left(1-p_{i p}\right)}}\right)
\end{gathered}
$$




$$
P_{\text {miss }} \simeq 1-Q\left(\frac{T_{2}-n p_{a p}}{\sqrt{n p_{a p}\left(1-p_{a p}\right)}}\right) \simeq 0.5+0.5 \operatorname{erf}\left(\frac{T_{2}-n p_{a p}}{\sqrt{2 n p_{a p}\left(1-p_{a p}\right)}}\right)
$$

The threshold $T_{2}$ can be fixed in the same way as that of $T_{1}$, based on the Bayesian criterion, as

$$
T_{2}=\frac{p\left(H_{0}\right)\left(C_{10}-C_{00}\right)}{p\left(H_{1}\right)\left(C_{01}-C_{11}\right)}
$$

where $p\left(H_{0}\right)$ and $p\left(H_{1}\right)$ are the a priori probabilities for inactive and active images, respectively, and $C_{00}$ and $C_{11}$ represent the costs incurred by making correct decisions, i.e, stating active and inactive images as active and inactive images, respectively. The elements $C_{10}$ and $C_{01}$ of the cost matrix represent the costs incurred by making wrong decisions, i.e, stating active and inactive images as inactive and active images, respectively.

Based on these calculations, if the number of active pixels exceeds the threshold $T_{2}$, then there is a significant change in the scene, and hence, the corresponding image is considered an active image; otherwise it is an inactive image. This decision test is a final test made at the sensor level, which tells whether a local change at the sensor has occurred or not. Assume the local decisions from all sensors as $S_{i}$, where $i=1,2, \ldots m$. These local decisions $S_{i}$ 's from individual sensors are relayed in the form of " 1 " or " 0 " to the base station through a wireless channel. Hence, by sending binary decisions, the data transmission between the sensor nodes and the base station is reduced greatly. The local decisions $S_{i}$ can now be written as

$$
S_{i}= \begin{cases}1, & \text { If } \lambda_{1} \geq T_{2} \text { consider as an active image } \\ 0, & \text { If } \lambda_{1}<T_{2} \text { consider as an inactive image }\end{cases}
$$




\subsection{Processing at the Fusion Center}

Assume that there are $m$ sensors deployed in the region of interest. Let all the $m$ sensors transmit their local decisions $S_{i}$ 's to the fusion center through an additive white Gaussian channel (AWGN), as depicted in the Figure.3.2. The fusion center receives noisy versions $Y_{i}$ 's of decisions $S_{i}$ 's sent by the sensors. This can be represented as

$$
Y_{i}=S_{i}+N_{i}
$$

where $N_{i} \sim \mathcal{N}\left(0, \sigma_{n}^{2}\right)$ and $i=\{1,2, \ldots m\}$. In order to detect incoming bits at the base station, a hypothesis test similar to the one used at the sensor level can be used at the base station also.

Let $p\left(Y_{i} \mid H_{0}\right)$ and $p\left(Y_{i} \mid H_{1}\right)$ represent the class conditional density functions of receiving bit " 0 " $\left(H_{0}\right)$ and receiving bit " 1 " $\left(H_{1}\right)$, respectively. In this thesis, we assume that both densities follow Gaussian distributions [28], which are defined as

$$
\begin{gathered}
p\left(Y_{i} \mid H_{0}\right)=\frac{1}{\sqrt{2 \pi \sigma_{n}^{2}}} \exp \left\{\frac{-Y_{i}^{2}}{2 \sigma_{n}^{2}}\right\} \\
p\left(Y_{i} \mid H_{1}\right)=\frac{1}{\sqrt{2 \pi \sigma_{n}^{2}}} \exp \left\{\frac{-\left(Y_{i}-1\right)^{2}}{2 \sigma_{n}^{2}}\right\}
\end{gathered}
$$

If $p\left(H_{0} \mid Y_{i}\right)$ and $p\left(H_{1} \mid Y_{i}\right)$ denote the a posteriori probabilities, the decision rule that maximizes the probability of correct decision, can be defined and stated as

$$
\frac{p\left(H_{1} \mid Y_{i}\right)}{p\left(H_{0} \mid Y_{i}\right)} \stackrel{H_{1}}{\stackrel{H_{0}}{\gtrless} 1} 1
$$

Equation (3.2.4) is called the maximum a posteriori (MAP) criterion. Using Baye's rule in equation (3.2.4), the decision rule can be rewritten as

$$
\frac{p\left(H_{1}\right) p\left(Y_{i} \mid H_{1}\right)}{p\left(H_{0}\right) p\left(Y_{i} \mid H_{0}\right)} \stackrel{H_{1}}{H_{0}} 1
$$


or

$$
\frac{p\left(Y_{i} \mid H_{1}\right)}{p\left(Y_{i} \mid H_{0}\right)} \stackrel{H_{1}}{\gtrless} \frac{p\left(H_{0}\right)}{H_{0}} \frac{\left.H_{1}\right)}{p\left(H_{1}\right.}
$$

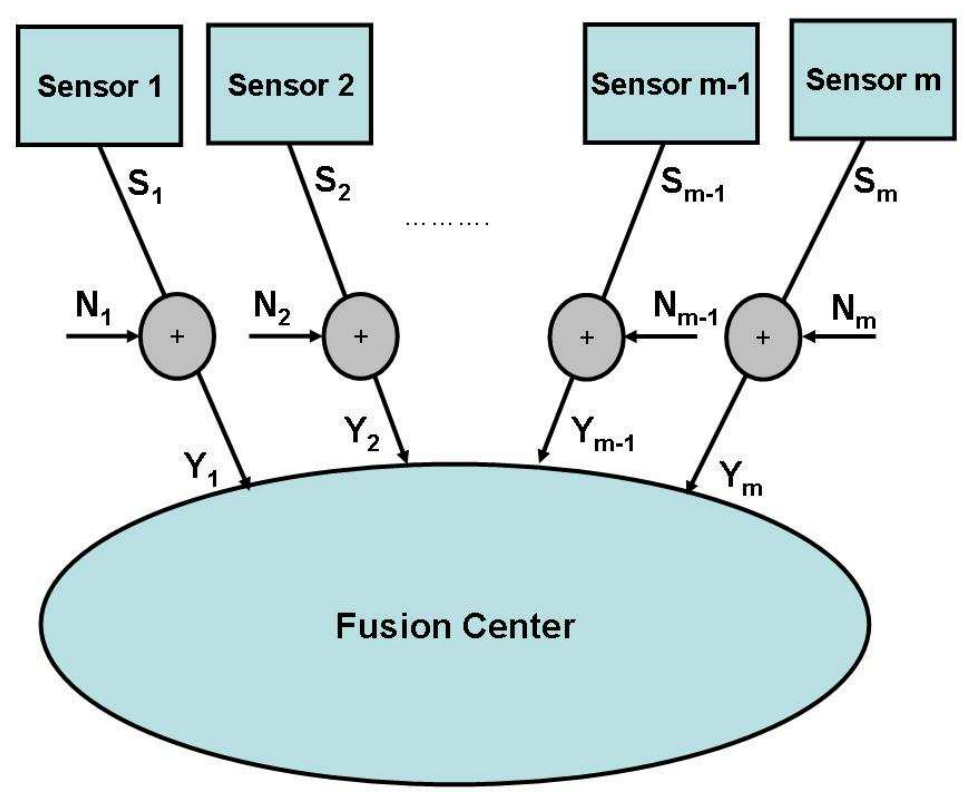

Figure 3.2: Information processing flow at fusion center. Local decisions from sensors $S_{i}$ are relayed to the base station via a wireless channel, which is prone to additive white gaussian noise $N_{i}$. The noisy versions of the local decisions are represented by $Y_{i}$. Before the fusion center makes a global decision, the MAP rule is used to detect the incoming bits. With these detected bits, a fusion rule is computed to make a global decision as to whether a change has taken place or not.

Based on equations (3.2.2) and (3.2.3), the likelihood ratio can be computed as

$$
L\left(Y_{i}\right)=\frac{p\left(Y_{i} \mid H_{1}\right)}{p\left(Y_{i} \mid H_{0}\right)}=e^{\left(2 Y_{i}-1\right) / 2 \sigma_{n}^{2}}
$$

where $L\left(Y_{i}\right)$ is called the likelihood statistic. The MAP decision rule consists of comparing this ratio with the constant $p\left(H_{0}\right) / p\left(H_{1}\right)$, which is called the decision 
threshold.

$$
T_{3}=\frac{p\left(H_{0}\right)}{p\left(H_{1}\right)}
$$

Equations (3.2.7) and (3.2.8) will lead to the formulation of a decision rule that helps in determining the final decision, which decides if a global change took place in the scene being monitored. The effect of a binary hypothesis decision rule is to divide the observation space into two regions $R_{0}$ and $R_{1}$, where $R_{0}=\left(-\infty, T_{3}\right)$ and $R_{1}=\left(T_{3}, \infty\right)$. If $Y_{i} \in R_{0}$, then a decision is made against $H_{0}$, which implies bit " 0 " is transmitted. Similarly, if $Y_{i} \in R_{1}$, then a decision is made against $H_{1}$, i.e, bit "1" is transmitted.

$$
\text { If } Y_{i} \in\left\{\begin{array}{l}
R_{0}, 0 \text { is transmitted } \\
R_{1}, 1 \text { is transmitted }
\end{array}\right.
$$

Once local decisions are detected at the fusion center, a fusion rule can be derived, which decides whether a global change has occurred or not. This is the final decision that is made at the system level. A statistic $\lambda_{2}$ that counts the number of "1"s received at the fusion center is defined as

$$
\lambda_{2}=\sum_{i=1}^{m} Y_{i}
$$

Assume the probabilities for change or detection are represented as $P_{c}$ and for no change or rejection as $P_{n c}$. At the fusion center, the probability of false alarm, denoted as $P_{f f a}$ and probability of miss denoted as $P_{f m i s s}$ are also calculated.

The probabilities for no change, change occurrence, false alarm, and miss can be written as $[26]$

$$
P_{n c}=P\left\{\lambda_{2}<T_{4} \mid H_{0}\right\}
$$




$$
\begin{gathered}
P_{c}=P\left\{\lambda_{2} \geq T_{4} \mid H_{1}\right\} \\
P_{f f a}=P\left\{\lambda_{2} \geq T_{4} \mid H_{0}\right\} \\
P_{\text {fmiss }}=P\left\{\lambda_{2}<T_{4} \mid H_{1}\right\}
\end{gathered}
$$

It can be shown that the sum follows Binomial distributions [7]. Let the probabilities of receiving bit "1" and bit "0" at the fusion center be represented as $p_{1}$ and $p_{0}$ respectively and are calculated as

$$
p_{1}=\lambda_{2} / m, \quad p_{0}=1-p_{0}
$$

Therefore, for a given threshold $T_{4}$, probabilities can be calculated as

$$
\begin{gathered}
P_{n c}=\sum_{i=0}^{T_{4}}\left(\begin{array}{c}
m \\
i
\end{array}\right) p_{0}^{i}\left(1-p_{0}\right)^{(m-i)} \\
P_{c}=\sum_{i=T_{4}}^{m}\left(\begin{array}{c}
m \\
i
\end{array}\right) p_{1}^{i}\left(1-p_{1}\right)^{(m-i)} \\
P_{f f a}=1-P_{n c}=1-\sum_{i=0}^{T_{4}}\left(\begin{array}{c}
m \\
i
\end{array}\right) p_{0}^{i}\left(1-p_{0}\right)^{(m-i)} \\
P_{f m i s s}=1-P_{c}=1-\sum_{i=T_{4}}^{m}\left(\begin{array}{c}
m \\
i
\end{array}\right) p_{1}^{i}\left(1-p_{1}\right)^{(m-i)}
\end{gathered}
$$

When $m$ is large enough, the probabilities in equations (3.2.16) to (3.2.19) can be calculated by using the Laplace-Demoivre approximation [27] as

$$
P_{n c} \bumpeq Q\left(\frac{-T_{4} p_{0}}{\sqrt{m p_{0}\left(1-p_{0}\right)}}\right) \bumpeq 0.5-0.5 \operatorname{erf}\left(\frac{-T_{4} p_{0}}{\sqrt{2 m p_{0}\left(1-p_{0}\right)}}\right)
$$




$$
\begin{gathered}
P_{c} \simeq Q\left(\frac{T_{4}-m p_{1}}{\sqrt{m p_{1}\left(1-p_{1}\right)}}\right) \simeq 0.5-0.5 \operatorname{erf}\left(\frac{T_{4}-m p_{1}}{\sqrt{2 m p_{1}\left(1-p_{1}\right)}}\right) \\
P_{f f a} \simeq 1-Q\left(\frac{-T_{4} p_{0}}{\sqrt{m p_{0}\left(1-p_{0}\right)}}\right) \simeq 0.5+0.5 \operatorname{erf}\left(\frac{-T_{4} p_{0}}{\sqrt{2 m p_{0}\left(1-p_{0}\right)}}\right) \\
P_{\text {fmiss }} \simeq 1-Q\left(\frac{T_{4}-m p_{1}}{\sqrt{m p_{1}\left(1-p_{1}\right)}}\right) \simeq 0.5+0.5 \operatorname{erf}\left(\frac{T_{4}-m p_{1}}{\sqrt{2 m p_{1}\left(1-p_{1}\right)}}\right)
\end{gathered}
$$

The threshold $T_{4}$ is also fixed using the Bayesian criterion as

$$
T_{4}=\frac{p\left(H_{0}\right)\left(C_{10}-C_{00}\right)}{p\left(H_{1}\right)\left(C_{01}-C_{11}\right)}
$$

Here, $p\left(H_{0}\right)$ and $p\left(H_{1}\right)$ represent the a priori probabilities for no change and change, respectively. The elements $C_{00}$ and $C_{11}$ of the cost matrix represent the costs incurred by making correct decisions. The elements $C_{10}$ and $C_{01}$ represent the costs incurred by making wrong decisions. Based on these calculations, if the number of "1"s exceeds the threshold $T_{4}$, then a global change is declared. 


\section{Chapter 4}

\section{Experimental Results and Analysis}

\subsection{Experimental Setup}

The performance of a decision support system can be evaluated by a set of metrics that includes the probability of detection and probability of false alarm. The tradeoffs between the probability of detection and probability of false alarm can be measured both at the sensor level and at the fusion center level using Receiver Operating Characteristic (ROC) curves.

The experimental setup shown in Figure 4.1 consisted of five image sensors (Cyclops cameras connected to motes) and a fusion center (mote connected to MIB510 programming board) connected to a desktop. The Cyclops camera was developed by the Center for Embedded Network Sensing at UCLA and Agilent Laboratories. This Cyclops camera is capable of taking both color and monochrome images with a resolution of $64 \times 64$ pixels. The motes and the MIB510 programming board were developed by Crossbow Technologies. This camera was connected to the wireless motes that acquire and transmit images at regular intervals to the base station over 
a wireless channel. The images received at the base station were viewed on a PC by making use of a Matlab code.

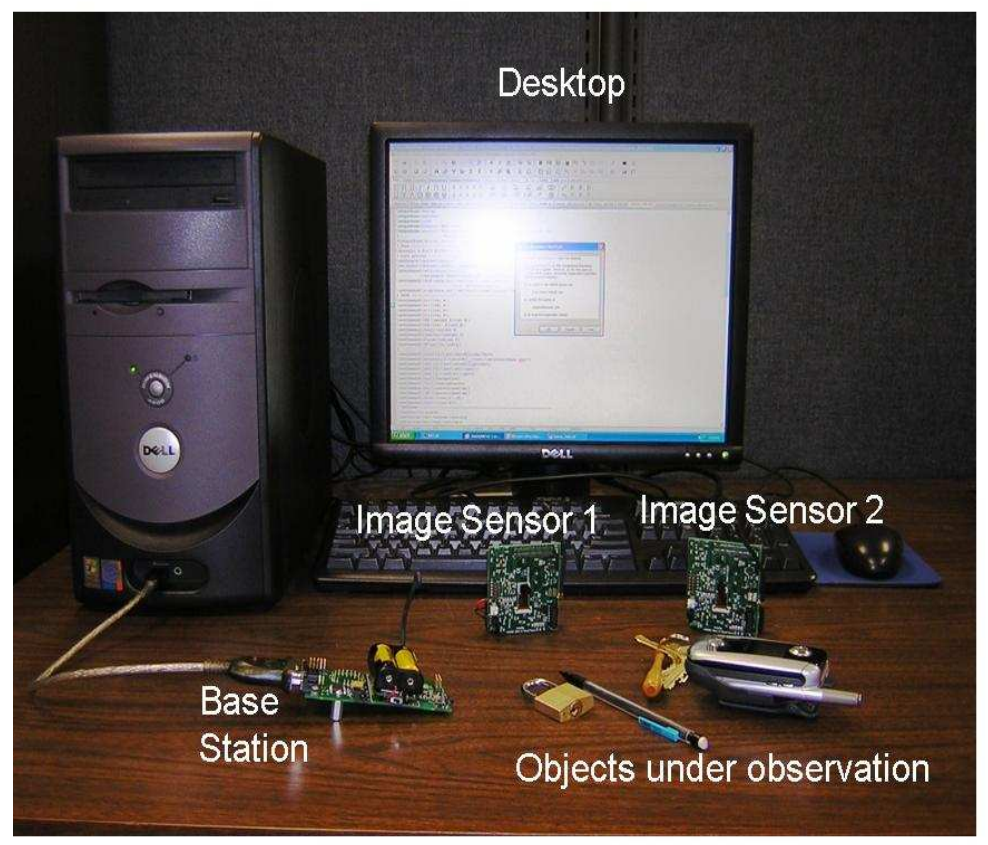

Figure 4.1: Experimental setup consisting of two image sensors (Cyclops camera) connected to crossbow motes, base station connected to the desktop through a serial to USB connector and objects under observation.

\subsection{Performance Analysis at the Sensor}

Five image sensors were placed randomly to monitor a particular region of interest consisting of several objects. Each sensor was allowed to monitor the same region in different directions. Also, each sensor took two consecutive images to detect changes. Two sets of images from two different sensors and their respective ROC curves are 
shown in Figures 4.2 to 4.5. The first image in each set is a normal view of the monitored area. The second image was taken after a change, such as appearance and disappearance of objects and motion of objects had been introduced. The change at a particular location can be significant or insignificant based on the sensor location and sensor observations. The illumination intensity is assumed to be constant throughout the experiment.

These sets of images were used for further processing. First, the difference between two consecutive images was found. The new image formed was called as the difference image. Processing at the sensor level mainly involved making two decisions. The first decision was to decide whether a pixel in the difference image is active or inactive. For this purpose a threshold $T_{1}$ was defined. After conducting several experiments, the threshold $T_{1}$ was set to 75 . The next step involved counting the number of active pixels in the difference image. Using this number, the probabilities for active pixels and inactive pixels were computed. The second decision was to decide whether the difference image is active or inactive. This was the final decision that is made at the sensor level. In order to make this decision, a second threshold $T_{2}$ was defined. The performance at the sensor level was analyzed using ROC curves. ROC curves were generated at the sensor level by fixing the first threshold $T_{1}$ and varying the threshold $T_{2}$ between 1 and 100. Different ROC curves were generated by using different thresholds. 

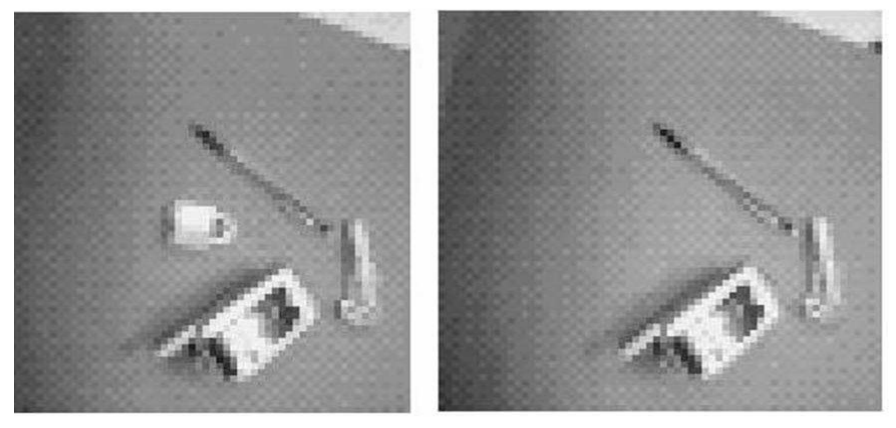

Figure 4.2: First set of images acquired by image sensor 1 . The image on the left consists of a few objects such as pen, cell phone, lock, and a set of keys. Some of the objects were removed before capturing the second image that is shown on the right.

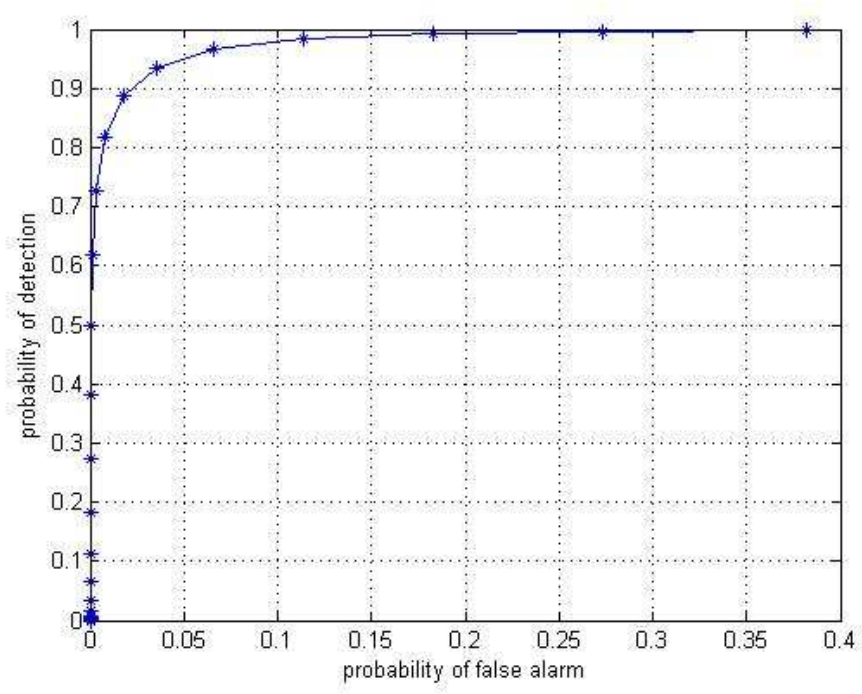

Figure 4.3: ROC curve at sensor 1, which was evaluated by using the set of images illustrated in Figure 4.2 with $T_{1}=75$ and $T_{2}$ varying between 1 and 100 . The probabilities for each choice of the threshold $T_{2}$ were generated theoretically from equations (3.1.12)and (3.1.17) to (3.1.20). 
Two sets of images taken from two different sensors are shown in Figures 4.2 and 4.4. In each set, the first image consists of several objects such as a pen, lock, cell phone, and a set of keys. Before taking the second image, some objects were removed. As shown, the lock was removed before taking the second picture. The first set of images were taken from a camera that is a small distance from the monitored area as opposed to the second set of images which were taken at a closer proximity. The ROC curves for each set of images are shown in Figures 4.3 and 4.5. The plots indicate that each choice of the threshold led to a pair of detection and false alarm rates somewhere on the ROC curve. Also, for any choice of the threshold, the detection rate was always higher than the false alarm rate, so the ROC curve is bowed upward. Another observation was that the change was significant for the second set of images, which were taken by the sensor closer to the region of interest. It can be implied that the closer the image sensor is to the region of interest, the better the resolution, resulting in a greater number of active pixels. Hence, $\lambda_{1}$ was higher in the second set of images compared to the first set of images. This resulted in improved change detection at the second sensor. 

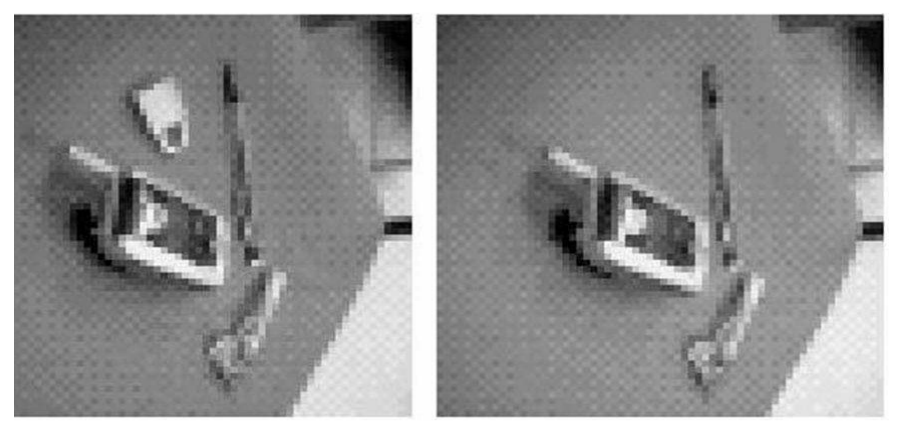

Figure 4.4: Second set of images acquired by sensor 2 placed at a different location and in a different direction. Notice that the images are taken from a closer proximity as opposed to the first set of images.

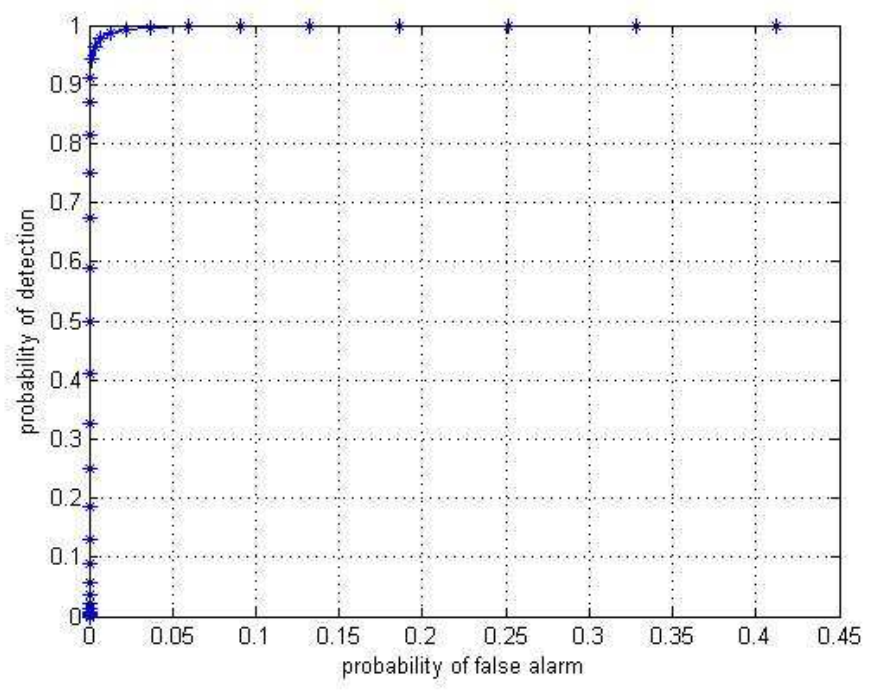

Figure 4.5: ROC curve at sensor 2, which was evaluated by using the set of images illustrated in Figure 4.2 with $T_{1}=75$ and $T_{2}$ varying between 1 and 100 . The probabilities for each choice of the threshold $T_{2}$ are generated theoretically from equations (3.1.12) and (3.1.17) to (3.1.20). 


\subsection{Performance Analysis at the Fusion Center}

The sensors send their local decisions in a binary form to the base station through an additive white Gaussian (AWGN) channel. Processing at the fusion center involved two steps. The first step was to detect the local decisions transmitted over the noisy channel. For this purpose, a threshold $T_{3}$ was defined at the fusion center to detect the binary local decisions transmitted from each sensor. After conducting several experiments, the threshold $T_{3}$ was set to 1.5 .

The second processing step involved making a global decision for change detection. For this purpose, the fourth threshold $T_{4}$ was defined. The performance of the system at the fusion center was evaluated by using ROC curve. This ROC curve is generated by fixing the third threshold $T_{3}$ and varying the fourth threshold $T_{4}$ from 1 to 5 . Three different types of curves were generated by considering two, three and four out

of five local detections, respectively. The plots for these three cases are illustrated in Figure 4.6. From the results it can be observed that, as the number of local detections increases, the detection rate increases to a great extent. 


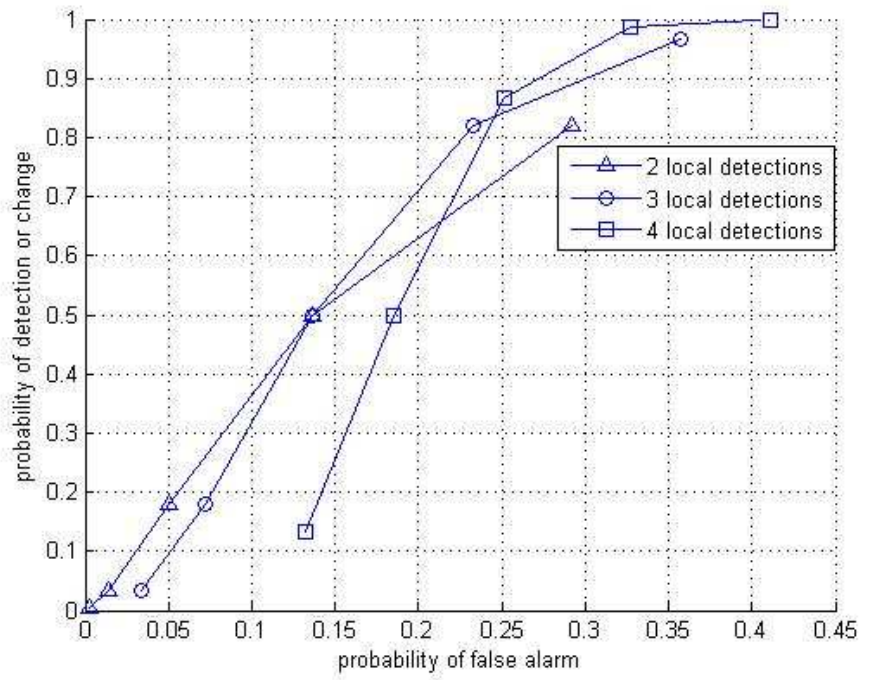

Figure 4.6: ROC curves at the fusion center with 2, 3 and 4 local detections from the sensors. The graphs are generated theoretically by making use of equations (3.2.15) and (3.2.20) to (3.2.23). 


\section{Chapter 5}

\section{Conclusion and Future Work}

\subsection{Conclusion}

In this thesis, a solution to a distributed image change detection problem in a wireless image sensor network was proposed. The system made use of four thresholds in order to make local and global decisions in the area being monitored. Two thresholds defined at the sensor level helped in making local decisions at the sensor level, and two thresholds defined at the fusion center helped in making global decisions about change in the scene that was being monitored.

\section{$5.2 \quad$ Future Work}

In this thesis, it was assumed that an additive white Gaussian noise channel corrupts local decisions transmitted from sensors. In the future, a system could be developed for real-world applications with more realistic models of channel noise. In this application, it was assumed that five sensors observed the region of interest, and the sensors did not communicate with their neighbors during local decision making. This work could be extended in the future by using more sensors, and also a system which considers the sensors communicating with their neighbors could be developed. 
REFERENCES 


\section{REFERENCES}

[1] R. J. Radke, S. Andra, O. Al-Kofahi, and B. Roysam, "Image Change Detection Algorithms: A Systematic Survey," IEEE Trans. on Image Processing, March 2005.

[2] R. Viswanath and P. K.Varshney, "Distributed Detection with Multiple Sensors: Part I-Fundamentals,” Proceedings of the IEEE, vol. 85, no.1, pp. 54-63, January 1997.

[3] M. Rahimi, "Cyclops: Image Sensing and Interpretation in Wireless Sensor Networks,” ACM Sensys Conference, November 2005.

[4] Crossbow Technology, http://www.xbow.com.

[5] D. Gay, P. Levis, R. von Behren, M. Welsh, E. Brewer, and D. Culler, "The nesC Language: A Holistic Approach to Networked Embedded Systems," In Proceedings of Programming Language Design and Implementation (PLDI), June 2003.

[6] “An Operating System for Networked Sensors,”http://www.tinyos.net.

[7] R. Niu, P. K.Varshney, M. Moore, and D. Klamer, "Decision Fusion in a Wireless Sensor Network with a Large Number of Sensors," Proc. 7th International. Conference on Information Fusion, Stockholm, Sweden, June 2004.

[8] R. Niu and P. K.Varshney, "Distributed Detection and Fusion in a Large Wireless Sensor Network of Random Size," EURASIP Journal on Wireless Communications and Networking, vol. 10, pp. 462-472, 2005.

[9] P. L. Rosin and E. Ioannidis, "Evaluation of Global Image Thresholding for Change Detection,” Pattern Recognition Letters, no. 24, pp. 23452356, January 2003.

[10] L. Bruzzone and D.F Prieto, "Automatic analysis of the Difference Image for Unsupervised Change Detection," IEEE Trans. Geoscience and Remote Sensing, vol. 38, pp. 1171-1182, May 2000.

[11] T. Kasetkasem and P. K.Varshney, "An Image Change Detection Algorithm Based on Markov Random Field Models," IEEE Trans. Geoscience and Remote Sensing, vol. 40, no. 8, pp.887-902, August 2002. 
[12] R. Collins, A. Lipton and T. Kanade, "Introduction to the special section on video surveillance," IEEE Trans. Pattern Anal. Mach. Intell, vol. 22, no. 8, pp. 745-746, August 2000.

[13] C. Stauffer and W. Grimson, "Learning patterns of activity using real-time tracking”, IEEE Trans. Pattern Anal. Mach. Intell, vol. 22, no. 8, pp.747757, August 2000.

[14] A. Hampapur, L. Brown, J. Connell, A. Ekin, N. Haas, M. Lu, H. Merkl, S. Pankanti, A. Senior, C.-F. Shu, and Y. L. Tian, "Smart Video Surveillance,” IEEE Signal Processing Magazine, pp. 38-51, March 2005.

[15] G. L. Foresti, C. Micheloni, L. Snidaro, P. Remagnino, and T. Ellis, “Active Video-Based Surveillance System,” IEEE Signal Processing Magazine, pp. 25-37, March 2005.

[16] M. Bosc, F. Heitz, J.-P. Armspach, I. Namer, D. Gounot, and L. Rumbach, "Automatic Change Detection in Multimodal Serial MRI: Application to Multiple Sclerosis Lesion Evolution," IEEE Trans. on Information Theory, July 2003.

[17] M. M. Kokar, J. A. Tomasik, and J. Weyman, "Data vs. Decision Fusion in the Category Theory Framework," 4th International Conference on Information Fusion, vol. 1, 2001.

[18] J.-F Chamberland and V. V. Veeravalli "Decentralized Detection in Wireless Sensor Networks with Dependent Observations," Proc. 2nd Intl. Conf. on Computing, Commun. and Contrl Technologies (CCCT04), Austin, TX, USA, August 2004.

[19] S. K. Jayaweera, "Decentralized Detection of Stochastic Signals in PowerConstrained Sensor Networks," IEEE Workshop on Signal Processing Advances in Wireless Communications (SPAWC), New York, June 2005.

[20] B. Chen and P. K.Varshney, "A Bayesian Sampling Approach to Decision Fusion Using Hierarchical Models,” IEEE Trans. on Signal Processing, vol. 50, no. 8, August 2002.

[21] R. S. Blum, S. A. Kassam, and H. V. Poor, "Distributed Detection with Multiple Sensors: Part II-Advanced Topics," Proceedings of the IEEE, vol.85, no.1, pp.64-78, January 1997.

[22] V. V. Veeravalli, "Decentralized Quickest Change Detection,” IEEE Trans. Information Theory, vol. 47, no. 4, pp. 1657-1665, May 2001. 
[23] P. Kulkarni, D. Ganesan, and P. Shenoy, "The Case for Multi-tier Camera Sensor Networks," Proceedings of the International Workshop on Network and Operating Systems Support for Digital Audio and Video, pp. 141 - 146, 2005.

[24] H.-M. Chen, S. Lee, R.M. Rao, M.-A. Slamani, and P.K. Varshney, "Imaging for Concealed Weapon Detection," IEEE Signal Processing Magazine, pp. 52-61, March 2005.

[25] C. B. Margi, R. Manduchi, and K. Obraczka, "Energy Consumption Tradeoffs in Visual Sensor Networks," Proceedings of 24th Brazilian Symposium on Computer Networks (SBRC 2006), June 2006.

[26] R. O. Duda and P. E. Hart and D. G. Stork, "Pattern Classification,” USA: Wiley-Interscience, 2001.

[27] A. Papoulis and S. Pillai, "Probability, Random Variable and Stochastic Processes", $4^{\text {th }}$ ed., McGraw Hill.

[28] S. K. Sam and A. M. Breipohl, "Random Signals Detection, Estimation and Data Analysis”, New York, USA: John Wiley, 1988. 